\title{
Evaluating X-Ray based Medical Imaging Devices with Fuzzy Preference Ranking Organization Method for Enrichment Evaluations
}

\author{
Dilber Uzun Ozsahin ${ }^{1}$ \\ Department of Biomedical Engineering, Near East University \\ Nicosia / TRNC Mersin 10 - Turkey, Gordon Center for Medical Imaging \\ Massachusetts General Hospital \& Harvard Medical School, Boston, U.S.A \\ Berna Uzun ${ }^{2}$ \\ Department of Mathematics \\ Near East University \\ Nicosia / TRNC Mersin 10 - Turkey \\ Musa Sani Musa ${ }^{3}$, Ilker Ozsahin ${ }^{4}$ \\ Department of Biomedical Engineering \\ Near East University \\ Nicosia / TRNC Mersin 10 - Turkey
}

\begin{abstract}
X-rays are ionizing radiation of very high energy, which are used in the medical imaging field to produce images of diagnostic importance. X-ray-based imaging devices are machines that send ionizing radiation to the patient's body, and obtain an image which can be used to effectively diagnose the patient. These devices serve the same purpose, only that some are the advanced form of the others and are used for specialized radiological exams. These devices have image quality parameters which need to be assessed in order to portray the efficiency, potentiality and negativity of each. The parameters include sensitivity and specificity, radiation dose delivered to the patient, cost of treatment and machine. The parameters are important in that they affect the patient, the hospital management and the radiation worker. Therefore, this paper incorporates these parameters into fuzzy PROMETHEE (Preference Ranking Organization Method for Enrichment Evaluation) multi-criteria decision theory in order to help the decision makers to improve the efficiency of their decision processes, so that they will arrive at the best solution in due course.
\end{abstract}

Keywords-X-ray-based imaging devices; medical imaging; fuzzy PROMETHEE

\section{INTRODUCTION}

Medical imaging refers to the use of ionizing and nonionizing radiation to produce images of the internal parts of the body for diagnostic purposes [1]. X-ray, which is the origin of medical imaging, was discovered in 1895 by Wilhelm Conrad Rontgen. Many medical imaging devices use X-rays to produce images of diagnostic value. The imaging devices include Computed Tomography (CT), Fluoroscopy, Conventional X-ray Machine, Mammography and Angiography [1]. These devices are similar because they all use X-ray radiation, but they differ in the type of radiological examination to be carried out. The devices have certain parameters like effective dose delivered to the patient, sensitivity, specificity, duration of scan, radiation dose, and cost. These parameters affect the patient directly, and it will be very important if they are analyzed in order for the parties concerned to have a good understanding of the procedures, their benefits and risks.

Making decision of using which technique for a specific patient or buying an x-ray based machine for a hospital can be difficult because of the high number of criteria and the range of their possible values. Fuzzy PROMETHEE is a method which gives the ability to compare selected alternatives according to some given criteria.

In this paper, Fuzzy PROMETHEE was used to evaluate the mostly used radiological equipment utilizing X-ray by using their parameters including radiation dose and scan time.

Section 1 of this paper introduces the paper while Section 2 introduces the various X-ray based medical imaging devices. In Section 3, the methodology and application is explained, Section 4 shows the result, and lastly Section 5 concludes the study.

\section{X-RAY BASED MEDICAL IMAGING DEVICES}

\section{A. Conventional X-Ray Machine}

This is the main device in most radiology departments, used to image internal structures. It is mainly dedicated to extremities, head, neck and abdominal region examinations. Indications include fractures of the limbs and skull, cervical spine and lumber vertebra exams, and abdomino-pelvic examinations. The main component of the X-ray machine is the Tube. The Tube consists of the anode, cathode, filament, glass envelope etc. The X-ray is produced when a current is passed through the filament, which results in heating the filament and electron production. These electrons are then accelerated to the anode target, where they become converted to X-ray and pass through a window to the object to be Xrayed. As the X-ray pass through the object, they hit a fluorescent cassette, which converts the X-ray into light, and a final image is produced after processing the cassette. X-ray imaging relies on the attenuation coefficient of the tissues and 
organs in the body. This is the reason why some parts appear bright while others appear dark [2].

\section{B. Angiography}

This is a specialized radiological examination that employs a contrast medium to outline the blood vessels, such as the arteries and the veins. It is mainly used to check the arterial or venous supply to the head, upper and lower limbs and thorax. Typical example of the procedure includes cerebral angiogram, pulmonary angiogram, coronary angiogram, etc.

The contrast is injected via a catheter into the femoral artery or vein, or into the brachial artery or vein. Several X-ray images of the area of interest are acquired and stored in films or digital format on computers. The procedure is usually indicated for aneurysm, stenosis and coronary artery disease [3].

\section{Computed Tomography $(C T)$}

This is a technique in which tomographic images are produced by transmitting $\mathrm{X}$-rays from an external source at several projection angles. This procedure is mostly used for anatomical localization and attenuation correction. CT uses a gantry containing the X-ray source and set of detectors, and also a bed where the patient lies during the examination. Axial images obtained from CT can be reformatted into coronal and sagittal, resulting to a $3 \mathrm{D}$ image set. CT is mostly used for tumors, brain and nervous system examination, urinary tract examination, and abdominal tumor [4].

\section{Fluoroscopy}

This imaging technique is used to obtain real-time images of a patient with the aid of a fluoroscope. It uses X-rays to obtain real-time (moving images) of the interval part of the body. Fluoroscope consists of a fluorescent screen with an Xray source between which the patient is placed. In modern fluoroscopes, the screen is connected to an X-ray beam condenser and a Charge - coupled device (CCD) video camera, which allows viewing or recording on an image monitor. Indications for fluoroscopy exams include special examinations like Hysterosalpingography (HSG), Intra-venous Urethrography (IVU), Barium studies, etc. Patient is exposed to a large amount of radiation, even if the dose used is minimal [5].

\section{E. Mammography}

This is a special device used to mage the breast. It has been very effective in the diagnosis of breast and axillary tumors. They use low energy X-ray to create images of the breast in both Medio-lateral and Cranio-caudal projections. These images are analyzed for any abnormal findings. Components include the tube, compression plate, cassette, breast support with grid, exposure button etc. Mammography has a limitation when it comes to women below the age of 30. It is suggested that women between the ages of 35-40 should undergo screening mammography at least once a year. During mammography procedure, the breast is slightly compressed between the two plates of the device for a few seconds. By so doing, all breast tissues are evaluated in 2 dimensions. 3D image can be obtained with digital breast tomosynthesis [6].

\section{METHODOLOGY AND APPLICATION}

The PROMETHEE method alone was proposed by [7] and [8]. The fuzzy PROMETHEE method allow the decision maker using fuzzy input data, which gives more flexibility to the decision maker while they compare alternatives in vague conditions. Application of this method was done by [9]-[15].

In the study conducted by [16], a detailed description of the fuzzy PROMETHEE method was given. In this study, we applied the same methodology for the evaluation of X-Ray based devices.

The most important parameters of the X-ray based medical imaging devices which include specificity, radiation dose, sensitivity, cost of devices and scan time are given in Table I as low bound, medium and upper. Choosing the parameters into three classes of low bound, medium and upper was done in order to apply triangular fuzzy numbers since the parameters of the alternatives are not crisp.

TABLE I. PARAMETERS OF THE DEVICES

\begin{tabular}{|l|l|l|l|l|l|}
\hline Unit & k\$ & Min. & \% & \% & mSv \\
\hline & Cost & Time & Specificity & Sensitivity & $\begin{array}{l}\text { Radiation } \\
\text { Dose }\end{array}$ \\
\hline \multirow{3}{*}{ Fluoroscopy } & 10 & 30 & 81 & 65 & 1,5 \\
& 50 & 40 & 85 & 70 & 3 \\
& 120 & 45 & 90 & 75 & 7 \\
\hline \multirow{3}{*}{ CT } & 155 & 15 & 78 & 85 & 2 \\
& 182.5 & 20 & 80 & 90 & 7 \\
\hline \multirow{3}{*}{ Mammography } & 210 & 25 & 87 & 95 & 10 \\
\hline \multirow{3}{*}{ X-Ray } & 50 & 8 & 69 & 79 & 0,2 \\
& 105 & 10 & 91 & 82 & 0,4 \\
& 99 & 5 & 97 & 95 & 0,7 \\
\hline \multirow{3}{*}{ Angiography } & 113 & 10 & 70 & 90 & 0,04 \\
& 125 & 15 & 75 & 93 & 0,1 \\
& 140 & 58 & 94 & 95 & 4,5 \\
\hline & 200 & 60 & 95 & 95 & 9,9 \\
& 62 & 96 & 96 & 15,8 \\
\hline
\end{tabular}

We used normalization and then we transform them to the triangular fuzzy numbers $(\mathrm{N}, \mathrm{a}, \mathrm{b})$ as shown in Table II.

TABLE II. TRIANGULAR FUZZY NUMBERS

\begin{tabular}{|l|l|l|l|l|l|}
\hline Unit & $\$$ & Min. & \% & \% & mSv \\
\hline & Cost & Time & Specificity & Sensitivity & $\begin{array}{l}\text { Rad. } \\
\text { Dose }\end{array}$ \\
& & & & & \\
\hline & $\mathrm{N}$ & $\mathrm{N}$ & $\mathrm{N}$ & $\mathrm{N}$ & $\mathrm{N}$ \\
& $\mathrm{a}$ & $\mathrm{a}$ & $\mathrm{a}$ & $\mathrm{a}$ & $\mathrm{a}$ \\
& $\mathrm{b}$ & $\mathrm{b}$ & $\mathrm{b}$ & $\mathrm{b}$ & $\mathrm{b}$ \\
\hline \multirow{4}{*}{ Fluoroscopy } & 0,084 & 0,29 & 0,20 & 0,16 & 0,15 \\
& 0,06 & 0,03 & 0,01 & 0,01 & 0,05 \\
& 0,07 & 0,01 & 0,00 & 0,00 & 0,05 \\
\hline \multirow{3}{*}{ CT } & 0,306 & 0,14 & 0,19 & 0,21 & 0,34 \\
& 0,03 & 0,01 & 0,01 & 0,00 & 0,08 \\
& 0,03 & 0,01 & 0,01 & 0,00 & 0,06 \\
\hline \multirow{3}{*}{ Mammography } & 0,134 & 0,07 & 0,22 & 0,19 & 0,02 \\
& 0,02 & 0,00 & 0,04 & 0,00 & 0,01 \\
& 0,00 & 0,02 & 0,00 & 0,02 & 0,00 \\
\hline \multirow{3}{*}{ X-Ray } & 0,190 & 0,07 & 0,17 & 0,22 & 0,00 \\
& 0,03 & 0,03 & 0,00 & 0,00 & 0,00 \\
\hline \multirow{3}{*}{ Angiography } & 0,03 & 0,02 & 0,00 & 0,01 & 0,04 \\
\hline & 0,285 & 0,43 & 0,226 & 0,22 & 0,485 \\
& 0,023 & 0,071 & 0,018 & 0,007 & 0,03 \\
& 0,022 & 0,046 & 0,01 & 0,01 & 0,033 \\
\hline
\end{tabular}


And then we used Yager index to see the magnitude of the parameters of the alternatives, which are fuzzy numbers. The linguistic scale of the importance rating of the parameters used for this application can be seen in Table III. It gives us information about the most important parameters to be compared among the alternatives. This information was obtained from expert in the medical imaging field.

TABLE III. LINGUISTIC SCALE FOR IMPORTANCE

\begin{tabular}{|l|l|l|}
\hline $\begin{array}{l}\text { Linguistic scale for } \\
\text { evaluation }\end{array}$ & $\begin{array}{l}\text { Triangular fuzzy } \\
\text { scale }\end{array}$ & $\begin{array}{l}\text { Importance ratings of } \\
\text { criteria }\end{array}$ \\
\hline Very high (VH) & $(0.75,1,1)$ & $\begin{array}{l}\text { Specificity, Radiation } \\
\text { dose, Sensitivity }\end{array}$ \\
\hline Important (H) & $(0.50,0.75,1)$ & Cost \\
\hline Medium (M) & $(0.25,0.50,0.75)$ & Scan Time \\
\hline Low (L) & $(0,0.25,0.50)$ & \\
\hline Very low(VL) & $(0,0,0.25)$ & \\
\hline
\end{tabular}

Furthermore, we used Yager index one more time in order to count the weight of the parameters. Lastly, we applied to the visual PROMETHEE program and used Gaussian preference function for the selected criteria (see Table IV). The best alternatives are expected to be of lower cost, have minimum radiation dose and scan time. They are also expected to have maximum specificity and sensitivity for the performance of the devices.

TABLE IV. VISUAL PROMETHEE APPLICATION

\begin{tabular}{|c|c|c|c|c|c|}
\hline Criteria & $\begin{array}{c}\text { Cost of } \\
\text { Dev. }\end{array}$ & $\begin{array}{c}\text { Scan } \\
\text { Time }\end{array}$ & Specificity & Sensitivity & $\begin{array}{c}\text { Rad. } \\
\text { dose }\end{array}$ \\
\hline Unit & $\$$ & Min. & $\%$ & $\%$ & $m S v$ \\
\hline Preferences & & & & & \\
\hline (min/max) & min & min & max & max & min \\
\hline Weight & 0,75 & 0,50 & 0,92 & 0,92 & 0,92 \\
\hline Preference Fn. & Gauss & Gauss & Gauss & Gauss & Gauss \\
\hline Evaluations & & & & & \\
\hline Fluoroscopy & 0,088 & 0,279 & 0,1992 & 0,1615 & 0,15 \\
\hline CT & 0,305 & 0,142 & 0,1877 & 0,2085 & 0,33 \\
\hline Mammography & 0,128 & 0,078 & 0,2045 & 0,1964 & 0,02 \\
\hline X-Ray & 0,189 & 0,069 & 0,1661 & 0,2184 & 0,02 \\
\hline Angiography & 0,285 & 0,42 & 0,2228 & 0,2222 & 0,49 \\
\hline
\end{tabular}

\section{RESUlTS AND DISCUSSION}

Table V shows the complete ranking of the X-ray based medical imaging devices when they were compared without including the cost of the machine. This is done in order to include parameters that are specific to the patient, being the receiver of the treatment. The total raking shows us that X-ray and mammography which gives less dose of radiation have relatively the same ranking. They are followed by fluoroscopy which all has a positive Net Flow. On the other hand, Computed Tomography and Angiography both having negative Net Flow came second to the last and last respectively on the total ranking table. It can be seen that this is due to the high dose of radiation they give to the patient.
TABLE V. COMPLETE RANKING OF THE IMAGING DEVICES With COST OF DEVICE DE-ACTIVATED

\begin{tabular}{|c|c|c|c|c|}
\hline $\begin{array}{c}\text { Complete } \\
\text { Ranking }\end{array}$ & $\begin{array}{c}\text { X-Ray Based } \\
\text { Devices }\end{array}$ & $\begin{array}{c}\text { Positive } \\
\text { outranking } \\
\text { flow }\end{array}$ & $\begin{array}{c}\text { Negative } \\
\text { outranking } \\
\text { flow }\end{array}$ & $\begin{array}{c}\text { Net } \\
\text { Flow }\end{array}$ \\
\hline 1 & X-Ray & 0,0017 & 0,0000 & 0,0017 \\
\hline 2 & Mammography & 0,0017 & 0,0000 & 0,0017 \\
\hline 3 & Fluoroscopy & 0,0006 & 0,0004 & 0,0002 \\
\hline 4 & CT & 0,0003 & 0,0009 & $-0,0006$ \\
\hline 5 & Angiography & 0,0000 & 0,0030 & $-0,0030$ \\
\hline
\end{tabular}

Table VI shows the complete ranking of the devices with cost of device activated. As it can be seen, there is no difference from the ranking while the cost was de-activated. But a slight change is noticed in the Net flow of Mammography and Conventional X-ray device. The idea to include the cost in Table VI was to produce a ranking table which will be useful for the hospital management, because price of device largely affects their selection process.

TABLE VI. COMPLETE RANKING OF THE IMAGING DEVICES WITH COST OF DEVICE ACTIVATED

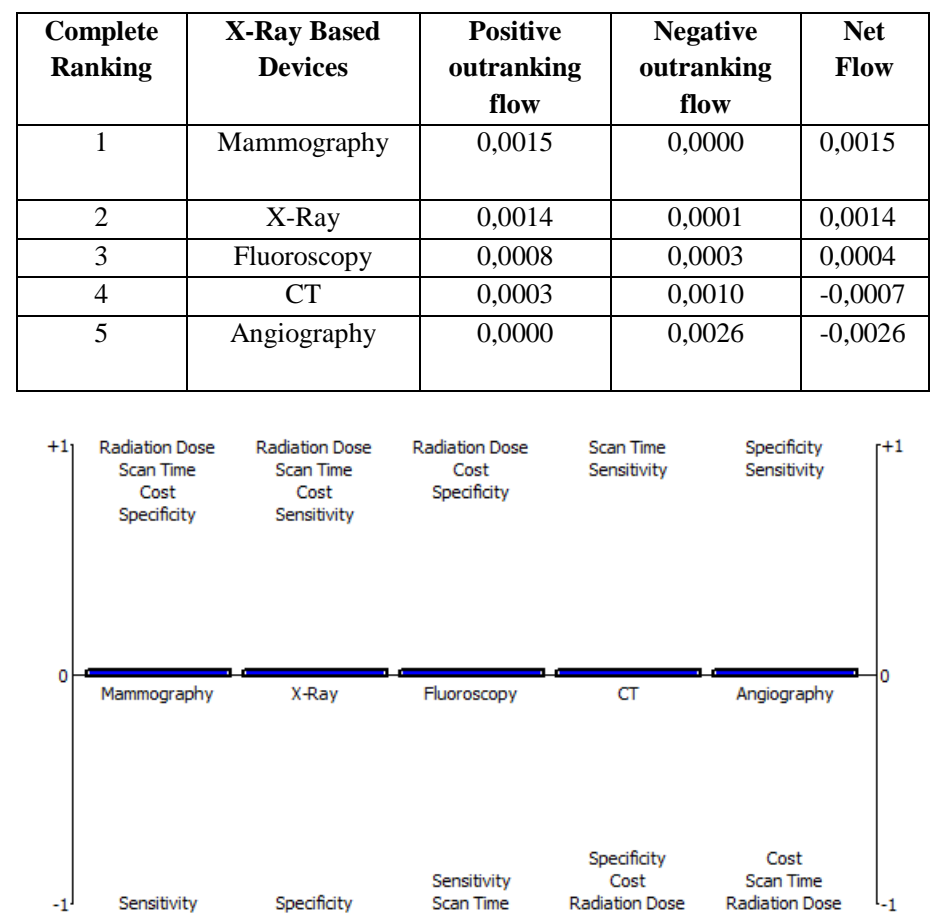

Fig. 1. PROMETHEE evaluation result.

The advantages and disadvantages according to the parameters of the devices are given in Fig. 1. These results also give very important information about the properties of the devices should incase the manufacturer needs to make any improvement for the specific property. This was obtained from the Decision Lab visual PROMETHEE program. The program gives the user the ability to manipulate the parameters and make further comparisons according to the decision maker preferences. 


\section{CONCLUSION}

Utilizing multi-criteria decision methods like fuzzy PROMETHEE gives the possibility to accomplish great outcomes by incorporating fuzzy data. This method was applied on various $\mathrm{X}$-ray based medical imaging devices and a useful result was obtained as shown in Section 4. The method has been tested and validated in previous studies, and it provides useful information when used in the present study. It is good and provides alternative solutions to decision maker. The concerned parties can rely on the outcome of this study to make the necessary decision in due course. The study can be improved by adding more criteria to the alternatives.

Furthermore, compared to other decision-making methods, the PROMETHEE method has proven to be efficient in many fields. The patients and the hospital management are the beneficiary of the outcomes of this study. This method can be applied to other decision-making problems that exist in medical imaging or other field of study.

\section{REFERENCES}

[1] Medical imaging. (2018). World Health Organization. Retrieved 22 March 2018.

[2] S. Nie, Z. Wang, W. Liu, and X. Liang, (2013). "Clinical Applications of X-ray, B-scan, and CT in the Diagnosis of Ocular Foreign Bodies," Eye Sciences. Vol. 28, no. 1, pp. 11-14, 2013.

[3] J. Yu, and J. Cockburn, “Angiography”. Retrieved 22 December 2017.

[4] C. Garvey, "Computed tomography in clinical practice," BMJ, vol. 324, no. 7345, pp. 1077-1080, 2002.

[5] S. Balter, J.W. Hopewell, J. D.L. Miller, L.K. Wagner, and M.J. Zelefsky, "Fluoroscopically Guided Interventional Procedures: A Review of Radiation Effects on Patients' Skin and Hair," Radiology, vol. 254, no. 2, pp. 326-341, 2010.

[6] F. Gilbert, S. Astley, A. Dibden, A. Seth, J. Morel, S. Bundred, et al., "Does Reader Performance with Digital Breast Tomosynthesis Vary according to Experience with Two-dimensional Mammography?," Radiology, vol. 283, no. 2, pp. 371-380, 2017.

[7] J.P. Brans, and P. Vincle, "A preference ranking organization method", Management Science,” vol. 31, no. 6, pp. 647-656, 1985.

[8] J.P. Brans, P. Vincke, and B. Mareschal, "How to select and how to rank projects: the PROMETHEE method," European Joirnal of Operational Research, vol. 24, pp. 228-238, 1986.

[9] M., Goumas, and V. Lygerou, "An extension of the PROMETHEE method for decision making in fuzzy environment: ranking of alternative energy exploitation projects," European Journal of Operational Research, vol. 123, pp. 606-613, 2000.

[10] J. Geldermann, T. Spengler, O. Rentz, "Fuzzy outranking for environmental assessment. Case study: iron and steel making industry," Fuzzy Sets and Systems, vol. 115, no. 1, pp. 45-65, 2000.SÉPS:

[11] R.U. Bilsel, G. Buyukozkan, D. Ruan, "A fuzzy preference ranking model for a quality evaluation of hospital web sites," International Journal of Intelligent System, vol. 21, no. 11, pp. 1181-1197, 2006.

[12] W.C. Chou, W.T. Lin, C.Y. Lin, "Application of fuzzy theory and PROMETHEE technique to evaluate suitable eco-technology method: a case study in Shismen reservoir watershed," Ecological Engineering, vol. 31, pp. 269-280, 2007. 'is

[13] G. Tuzkaya, B., Gülsün, C. Kahraman, D. Özgen, “An integrated fuzzy multi-criteria decision making methodology for material handling equipment selection problem and an application," Expert Systems Applications, vol. 37, no. 4, pp. 2853-2863, 2010.

[14] A. Ozgen, G. Tuzkaya, U.R. Tuzkaya, D. Ozgen, "A Multi-Criteria Decision Making Approach for Machine Tool Selection Problem in a Fuzzy Environment," International Journal of Computer Intelligence

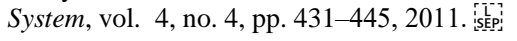

[15] D. Ozsahin, B. Uzun, M. Musa, N. Şentürk, F. Nurçin, and I. Ozsahin, "Evaluating nuclear medicine imaging devices using fuzzy PROMETHEE method," Procedia Computer Science, vol. 120, pp. 699$705,2017$.

[16] D. Uzun, B. Uzun, M. Sani, A. Helwan, C. Nwekwo, and F. Veysel, F. et al., "Evaluating Cancer Treatment Alternatives using Fuzzy PROMETHEE Method," International Journal of Advanced Computer Science and Applications, vol. 8, no. 10, pp. 177-182, 2017. 